\title{
Incidencia de Accidentes de Trabajo Declarados en Ecuador en el Período 2011-2012
}

\author{
INCIDENCE OF ACCIDENTS REPORTED IN ECUADOR IN 2011-2012
}

\author{
Antonio R. Gómez Garcia', Pablo R. Suasnavas Bermúdez ${ }^{2}$ \\ 1. Gerente de Administración de Riesgos Laborales de Biodimed. Quito, Ecuador. Profesor de la Facultad de Seguridad y Salud Ocupacional, Universidad \\ Internacional SEK, Ecuador. \\ 2. Gerente de SafeWork. Quito, Ecuador. Profesor de la Facultad de Seguridad y Salud Ocupacional, Universidad Internacional SEK, Ecuador.
}

\begin{abstract}
RESUMEN
Introducción: el conocimiento de la incidencia de los accidentes de trabajo es un aspecto fundamental para conocer y adoptar políticas gubernamentales para reducir la siniestralidad laboral. Existen razones para pensar que en Ecuador preexiste una infraestimación de la declaración y registro de accidentes de trabajo.

Objetivos: la información y análisis de la incidencia de los accidentes de trabajo permite conocer la siniestralidad laboral de un país y formular acciones encaminadas a mejorar las condiciones de trabajo. En la actualidad no existen datos estadísticos sobre el índice de incidencia en Ecuador que permitan la comparación homogénea y estadística con otros países. El objetivo del presente estudio es describir los accidentes de trabajo registrados en el Instituto Ecuatoriano de Seguridad Social en los períodos de 2011 y 2012 y calcular su correspondiente índice de incidencia.

Métodos: estudio descriptivo y transversal. La fuente de información analizada de los accidentes de trabajo se obtuvo a través de la Subdirección de Prevención de Riesgos y Control de Prestaciones del IESS. Para el análisis se establecieron las variables: provincia, actividad económica, género, tipo de lesión, ubicación, consecuencias, día, hora y tipo de incapacidad del accidente de trabajo. Se establecieron como indicadores porcentajes e índices de incidencia.

Resultados: las notificaciones de accidentes de trabajo registrados en 2012 representan una incidencia de 550,5 casos por cada por 100.000 trabajadores, indice superior al registrado en 2011 con 419,7. La incidencia de los accidentes de trabajo que derivan en una incapacidad temporal en 2012 es de 527 por cada 100.000 trabajadores.

Conclusiones: con la entrada en vigor de la Resolución C.D. No. 390Reglamento del Seguro General de Riesgos del Trabajo de 2011- se han incrementado el número de notificaciones de accidentes de trabajo. La industria manufacturera es la de mayor índice de incidencia. Las principales provincias del país con mayor índice de incidencia son Pichincha, con 371,1 casos por cada 100.000 trabajadores, y 1037,7 en la provincia del Guayas en 2012.
\end{abstract}

(Gómez A, Suasnavas P, 2015. Incidencia de Accidentes de Trabajo Declarados en Ecuador en el Período 2011-2012. Cienc Trab. Ene-Abr; 17 [52]: 49-53).

Palabras clave: DECLARACIONES, ACCIDENTES DE TRABAJO, ÍNDICE DE INCIDENCIA, ECUADOR.

Correspondencia / Correspondence:

Antonio R. Gómez García

Facultad de Seguridad y Salud Ocupacional. Campus Miguel de Cervantes

Alberto Einstein, s/n y 5ta transversal, Carcelén, Quito. Ecuador

Tel: (593 2) 3974800 Ext. 119 • Móvil: 0987609222 • Fax: (593 2) 2485105

e-mail: antonio.gomez@uisek.edu.ec

Recibido: 11 de Diciembre de 2014 / Aceptado: 18 de Enero 2015

\section{ABSTRACT}

Introduction: knowledge of the incidence of accidents at work is a key aspect to understand and adopt government policies to reduce workplace accidents. There are reasons to believe that in Ecuador preexists an underestimation of the declaration and registration of accidents. Objectives: The information and analysis of the incidence of occupational accidents allows to know a the workplace accidents of a country and make efforts to improve working conditions. Currently there are no statistics on the incidence rate in Ecuador that allow homogeneous and statistical comparison with other countries. The aim of this study is to describe the accidents recorded in the Ecuadorian Institute of Social Security in the periods 2011 to 2012 and calculate its corresponding incidence rate.

Methods: It is a cross-sectional study. The source of information analyzed accidents at work was obtained through the Office of Risk Prevention and Control Features IESS. To analyze the next variables were established: province, economic activity, gender, type of injury, location, consequences, day, time and type of disability accident. They were established as percentages indicators and incidence rates.

Results: notifications of accidents recorded in 2012 represent an incidence of 550,5 cases per 100,000 workers, more than in 2011 with 419,7 index. The incidence of occupational accidents which result in temporary incapacity in 2012 is 527 per per 100.000 workers.

Conclusions: With the entry into force of Resolution CD No. 390 Regulation of the Seguro General de Riesgos del Trabajo de 2011 have increased the number of notifications of accidents. Manufacturing is a key indicator of incidence. The main provinces with the highest incidence are Pichincha with 371,1 cases per 100.000 workers and 1037,7 in the province of Guayas in 2012.

Key words: DECLARATIONS, ACCIDENTS, INCIDENCE RATE, ECUADOR.

\section{INTRODUCCIÓN}

En la actualidad se emplean diferentes indicadores en siniestralidad laboral a nivel latinoamericano cuyo objetivo reside en obtener información sobre los accidentes de trabajo y enfermedades profesionales a fin de establecer programas eficaces de seguridad y salud ocupacional. ${ }^{1-2}$ 
Con esta premisa, la I Estrategia Iberoamericana de Seguridad y Salud en el Trabajo para el periodo 2010-20131, propuso la mejora de los sistemas de información y registro de los accidentes de trabajo y enfermedades profesionales con el objeto de armonizar entre los países registros de siniestralidad laboral.

Según la $\mathrm{OIT}^{3}$, el índice de incidencia de accidentes de trabajo se obtiene como un cociente donde el numerador se corresponde con el número de accidentes de trabajo multiplicado por cien mil y el denominador es la media anual de los trabajadores afiliados a la seguridad social. Generalmente se multiplica por cien mil; no obstante, en otros países se realiza el cálculo por cada 1.000 , por cada 100.000 ó incluso por cada 200.000 trabajadores afiliados. ${ }^{3-5}$

Desde 2010, Ecuador dispone de una normativa ${ }^{6-7}$ que propicia la incorporación y registro de datos sobre siniestralidad laboral a nivel nacional, además de otras disposiciones reglamentarias. ${ }^{8-10}$

Asimismo, los datos de siniestralidad laboral publicados por el Instituto Ecuatoriano de Seguridad Social de Ecuador carecen de un cálculo de incidencia, lo cual impide la comparación con otros países de Latinoamérica ${ }^{11}$ e internacionales $y$, por tanto, ajustarse a un sistema armonizado de indicadores básicos de siniestralidad y salud laboral en Iberoamérica. ${ }^{1}$ Hecho constatado en el informe publicado en 2011 por el Instituto de Salud y Trabajo (ISAT) -Diagnóstico Situacional en Seguridad y Salud en el Trabajo en Ecuador-, que indica textualmente que: "no se cuenta con estudios nacionales sobre este problema, por lo que es dificil hacer aproximaciones a la magnitud del mismo".

La conceptualización de accidente de trabajo ${ }^{7}$ se define como todo suceso imprevisto y repentino que ocasione al afiliado lesión corporal o perturbación funcional, o la muerte inmediata o posterior, con ocasión o como consecuencia del trabajo que ejecuta por cuenta ajena. Para efectos de la concesión de la prestación del accidente de trabajo al afiliado ${ }^{12}$, se considera accidente de trabajo el que se produjere en el lugar de trabajo o fuera de él, que ocurriere en la ejecución de trabajo a órdenes del empleador, el que ocurriere por la acción de terceras personas y/o el que sobreviniere durante las pausas o interrupciones de las labores, igualmente en actividades gremiales o sindicales y los accidentes in-itínereo en tránsito; "cuando el recorrido se sujete a una relación cronológica de inmediación entre las horas de entrada y salida del domicilio al trabajo y viceversa". ${ }^{7}$

El empleador no sólo está obligado a enviar anualmente a las unidades provinciales del Seguro General de Riesgos del Trabajo de IESS los Índices Reactivos (Frecuencia, Gravedad y Tasa de Riesgo), sino también a notificar en un plazo máximo de 10 días la ocurrencia del accidente de trabajo a dichas unidades provinciales. La información registrada en el Seguro General de Riesgos del Trabajo permite conocer el número de accidentes de trabajo en Ecuador.

El objetivo del presente estudio es describir los accidentes de trabajo y calcular su correspondiente índice de incidencia en los períodos de 2011 y 2012. La justificación de establecer los períodos de análisis objeto de estudio reside en la ausencia de datos oficiales por parte de los organismos públicos para años posteriores.

\section{MATERIAL Y MÉTODOS}

El presente estudio es de tipo descriptivo y transversal. Se analiza la información registrada en las notificaciones de accidentes de trabajo facilitados por la Subdirección de Prevención de Riesgos y Control de Prestaciones del Instituto Ecuatoriano de Seguridad Social en formato electrónico para los períodos de 2011 y 2012; la consulta de información se obtuvo a través de los Boletines Estadísticos publicados periódicamente por la Dirección Actuarial del IESS. Las variables de interés ${ }^{1}$ fueron establecidas según los reportes de accidentes de trabajo del IESS: Provincia (Azuay, Bolivar, Guayas, Pichincha, etc.), Actividad Económica ${ }^{13}$, Género (masculino-femenino), Tipo del accidente, Ubicación de la lesión, Consecuencias (Fracturas, Traumatismos, Contusiones, etc.), Día de ocurrencia del accidente, Hora de ocurrencia del accidente y Tipo de incapacidad (Temporal, Permanente, Muerte, etc.).

Se empleó la estadística descriptiva y cálculo del índice de incidencia para cada una de las variables objeto de estudio. Para el cálculo de los índices de incidencia se utilizó como denominador la población afiliada $^{14}$ (en 2011 un total de 2.224.630 de trabajadores y 2.480.721 en 2012, respectivamente) al seguro social por 100.000 para cada período analizado, armonizados con la fórmula establecida por la Eurostat ${ }^{14}$, metodología empleada para los países pertenecientes a la Unión Europea y comparables para otros países internacionales.

\section{RESULTADOS}

En el año 2011 se notificaron en Ecuador un total de 9.338 accidentes de trabajo (A.T.) y 13.657 en 2012, suponiendo un incremento de 4.319 en el período analizado. El índice de incidencia (I.I.) para el período de 2011 fue de 419,76 y 550,53 en 2012, aumentándose en 130,77 .

Las provincias con mayor número de accidentes de trabajo registrados en 2011 corresponden a Guayas con 4.181 (44,77\%) y Pichincha con 2.005 (21,47\%). Para el año 2012, igualmente estas dos provincias encabezaron los notificaciones con 6.801 (49,80\%) en Guayas y $3.352(24,54 \%)$ en Pichincha. En la Tabla 1, se presentan los resultados tabulados.

Tabla 1.

Distribución de A.T. e I.I. por Provincia 2011-2012.

$\begin{array}{lcccccccc}\text { PROVINCIA } & \text { n A.T. } & \begin{array}{c}\text { \% n } \\ \text { A.T. }\end{array} & \begin{array}{c}\text { P.A. } \\ \text { IESS }\end{array} & \text { I.I. } & \text { n A.T. } & \begin{array}{c}\text { \% n } \\ \text { A.T. }\end{array} & \begin{array}{c}\text { P.A. } \\ \text { IESS }\end{array} & \text { I.I. } \\ \text { Azuay } & 437 & 4,7 \% & 126682 & 344,96 & 501 & 3,7 \% & 137282 & 364,94 \\ \text { Bolivar } & 45 & 0,5 \% & 13184 & 341,32 & 58 & 0,4 \% & 14349 & 404,21 \\ \text { Cañar } & 247 & 2,6 \% & 24477 & 1009,11 & 500 & 3,7 \% & 26133 & 1913,29 \\ \text { Carchi } & 34 & 0,4 \% & 14723 & 230,93 & 25 & 0,2 \% & 15798 & 158,25 \\ \text { Chimborazo } & 125 & 1,3 \% & 39030 & 320,27 & 172 & 1,3 \% & 43538 & 395,06 \\ \text { Cotopaxi } & 195 & 2,1 \% & 42100 & 463,18 & 279 & 2,0 \% & 45087 & 618,80 \\ \text { El Oro } & 150 & 1,6 \% & 70290 & 213,40 & 242 & 1,8 \% & 81854 & 295,65 \\ \text { Esmeraldas } & 136 & 1,5 \% & 38875 & 349,84 & 239 & 1,8 \% & 41415 & 577,09 \\ \text { Galápagos } & 8 & 0,1 \% & 5471 & 146,23 & 17 & 0,1 \% & 6205 & 273,97 \\ \text { Guayas } & 4181 & 44,8 \% & 594287 & 703,53 & 6801 & 49,8 \% & 655362 & 1037,75 \\ \text { Imbabura } & 85 & 0,9 \% & 44642 & 190,40 & 104 & 0,8 \% & 50235 & 207,03 \\ \text { Loja } & 120 & 1,3 \% & 45972 & 261,03 & 146 & 1,1 \% & 53245 & 274,20 \\ \text { Los Ríos } & 415 & 4,4 \% & 51483 & 806,09 & 0 & 0,0 \% & 58068 & 0,00 \\ \text { Manabi } & 287 & 3,1 \% & 126682 & 226,55 & 274 & 2,0 \% & 134287 & 204,04 \\ \text { Morona Santiago } & 42 & 0,4 \% & 10582 & 396,90 & 57 & 0,4 \% & 12344 & 461,76 \\ \text { Napo } & 37 & 0,4 \% & 9753 & 379,37 & 133 & 1,0 \% & 10945 & 1215,17 \\ \text { Orellana } & 85 & 0,9 \% & 11906 & 713,93 & 89 & 0,7 \% & 13543 & 657,17 \\ \text { Pastaza } & 29 & 0,3 \% & 9100 & 318,68 & 0 & 0,0 \% & 10386 & 0,00 \\ \text { Pichincha } & 2005 & 21,5 \% & 799628 & 250,74 & 3352 & 24,5 \% & 903084 & 371,17 \\ \text { Santa Elena } & 41 & 0,4 \% & 16766 & 244,54 & 123 & 0,9 \% & 19813 & 620,80 \\ \text { Santo Domingo } & & & & & & & & \\ \text { Tsáchilas } & 234 & 2,5 \% & 36444 & 642,08 & 201 & 1,5 \% & 40449 & 496,92 \\ \text { Sucumbios } & 178 & 1,9 \% & 15078 & 1180,53 & 145 & 1,1 \% & 18351 & 790,15 \\ \text { Tungurahua } & 183 & 2,0 \% & 68195 & 268,35 & 199 & 1,5 \% & 78013 & 255,09 \\ \text { Zamora Chinchipe } & 39 & 0,4 \% & 9280 & 420,26 & 0 & 0,0 \% & 10935 & 0,00 \\ \text { TOTAL } & 9338 & 100 \% & 2224630 & 419,76 & 13657 & 100 \% & 2480721 & 550,53\end{array}$

A.T.: accidentes de trabajo.

P.A.: personas afiliadas al Instituto Ecuatoriano de Seguridad Social.

I.I.: índice de incidencia. 
Figura 1.

Distribución de A.T. por Actividad Económica 2011-2012.

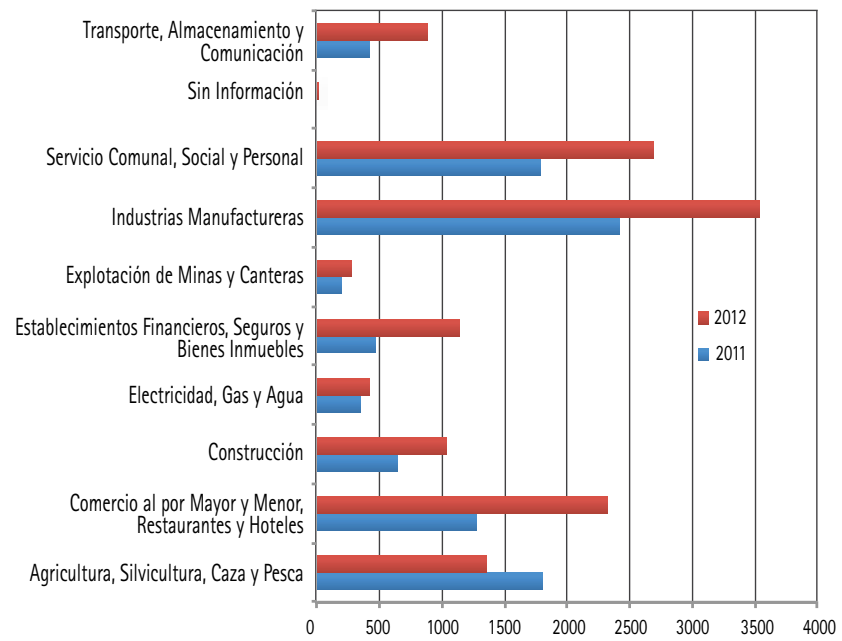

$\mathrm{Al}$ no disponer de datos oficiales y válidos sobre la distribución de la población afiliada por actividad económica se dificultó el cálculo del indice de incidencia. Las actividades económicas con mayor siniestralidad laboral la conformaron, para el año 2011: las Industrias Manufactureras con 2.415 (25,9\%) accidentes de trabajo notificados; Agricultura, Silvicultura, Caza y Pesca con 1.802 (19,3\%); Servicio Comunal, Social y Personal 1.785 (19,1\%), entre las actividades económicas de mayor siniestralidad laboral. En 2012, la distribución fue la siguiente: las Industrias Manufactureras con 3.536 (25,9\%) accidentes de trabajo; Servicio Comunal, Social y Personal con 2.692 (19,7\%); y, Comercio al por Mayor y Menor, Restaurantes y Hoteles con 2.319 (17\%) accidentes de trabajo. Figura 1.

Del mismo modo el índice de incidencia no se pudo establecer para género; en 2011 para el género masculino se notificaron un total de $7.971(85,4 \%)$ accidentes de trabajo y $1.367(14,6 \%)$ para el femenino. En 2012, para el género masculino se notificaron $11.122(81,4 \%)$ y femenino $2.535(18,6 \%)$.

El índice de incidencia de los accidentes de trabajo en función del tipo de lesión sobre la población afiliada para 2011 se distribuye en Comisión de Servicio I.I. 18,8. En el trabajo I.I. 336,1 e I.I. 64,9 En el trayecto (In-Itínere). Para 2012, Comisión de Servicio I.I. 23,9. En el trabajo I.I. 420,3, En el trayecto I.I. 106,0 y Sin información I.I. 0,3. En la Tabla 2 se presentan los índices de incidencia en función del tipo de lesión.

Respecto a la ubicación de la lesión de los accidentes de trabajo notificados, destacan las lesiones localizadas en los miembros superiores: 2011 con 3.346 (35,8\%) y 2012 con 4.873 (35,7\%) lesiones.Y, miembros inferiores: 2011 con 2.627 (28,1\%) y 2012 con $3.940(28,8 \%)$ lesiones.

Destacan en las consecuencias de la lesión de los accidentes de trabajo notificados los traumatismo superficiales con un I.I. de

Tabla 2.

Distribución de I.I. por Tipo de lesión 2011-2012.

$\begin{array}{lcccccc} & & 2011 & & & 2012 & \\ \text { TIPO } & \text { n A.T. } & \% \text { n A.T. } & \text { I.I. } & \text { n A.T. } & \% \text { n A.T. } & \text { I.I. } \\ \text { Comisión de Servicio } & 418 & 4,5 \% & 18,8 & 594 & 4,3 \% & 23,9 \\ \text { En el trabajo } & 7477 & 80,1 \% & 336,1 & 10427 & 76,3 \% & 420,3 \\ \text { En el trayecto } & 1443 & 15,5 \% & 64,9 & 2629 & 19,3 \% & 106,0 \\ \text { Sin información } & 0 & 0,0 \% & 0,0 & 7 & 0,1 \% & 0,3 \\ \text { TOTAL } & 9338 & 100 \% & 419,76 & 13657 & 100 \% & 550,53\end{array}$

A.T.: accidentes de trabajo. I.I.: índice de incidencia.
Tabla 3.

Distribución de I.I. por Consecuencias de la lesión 2011-2012.

\begin{tabular}{|c|c|c|c|c|c|c|}
\hline CONSECUENCIAS & n A.T. & $\begin{array}{c}2011 \\
\% \text { n A.T. }\end{array}$ & I.I. & n A.T. & $\begin{array}{l}2012 \\
\% \text { n A.T. }\end{array}$ & I.I. \\
\hline Fracturas y luxaciones & 1920 & $20,6 \%$ & 86,3 & 2861 & $20,9 \%$ & 115,3 \\
\hline as y esguinces & 768 & $8,2 \%$ & 34,5 & 1449 & $10,6 \%$ & 58,4 \\
\hline $\begin{array}{l}\text { ones y } \\
\text { smos internos }\end{array}$ & 424 & $4,5 \%$ & 19,1 & 418 & $3,1 \%$ & 16,8 \\
\hline enucl & 360 & $3,9 \%$ & 16,2 & 341 & $2,5 \%$ & 13,7 \\
\hline Otras & 1903 & 26 & 85,5 & 2502 & 18 & 100,9 \\
\hline os superficiales & 2141 & 22,9 & 96,2 & 2838 & 20,8 & 114,4 \\
\hline y aplastamientos & 972 & 16 & 43,7 & 1930 & 14,1 & 77,8 \\
\hline Quema & 364 & $3,9 \%$ & 16,4 & 444 & $3,3 \%$ & 17,9 \\
\hline $\begin{array}{l}\text { Envenenamiento e } \\
\text { intoxicaciones agudas }\end{array}$ & 42 & $0,4 \%$ & 1,9 & 57 & $0,4 \%$ & 2,3 \\
\hline $\begin{array}{l}\text { Efectos de exposición al frio y } \\
\text { a otros estados conexos }\end{array}$ & 8 & & 0,4 & 9 & & 0,4 \\
\hline Asfixia & 23 & & 1,0 & 33 & $0,2 \%$ & 1,3 \\
\hline $\begin{array}{l}\text { Efectos de electricidad } \\
\text { Efectos nocivos de }\end{array}$ & 31 & $0,3 \%$ & 1,4 & 21 & $0,2 \%$ & 0,8 \\
\hline $\begin{array}{l}\text { radiaciones } \\
\text { Múltiples y otras }\end{array}$ & 1 & $0,0 \%$ & 0,0 & 1 & $0,0 \%$ & 0,0 \\
\hline no definid & 381 & & 17,1 & & & 30,4 \\
\hline TOTAL & 9338 & $100 \%$ & 419,76 & 13657 & $100 \%$ & 550,53 \\
\hline
\end{tabular}

A.T.: accidentes de trabajo. I.I.: indice de incidencia.

Figura 2.

Distribución de I.I. por Hora 2011-2012.

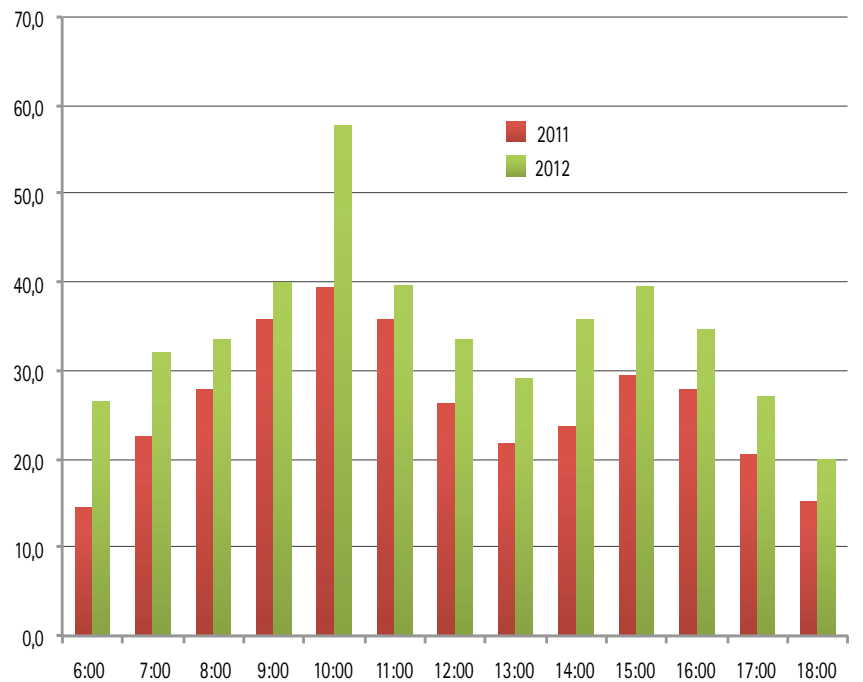

96.2 en 2011 e I.I. de 114.4 para 2012, seguido de las fracturas y luxaciones con un I.I. 86,3 en 2011 y 2012 con un I.I. de 115,3. Tabla 3.

El día de la semana donde mayor siniestralidad se registró en 2011 y 2012 fueron los martes. No existiendo diferencias significativas entre el día de la semana y número de accidentes de trabajo, el promedio de I.I. para 2011 se sitúa en 60,0 e I.I. 60,8 en 2012.

La franja horario donde mayor ocurrencia existió en los accidentes de trabajo se situó entre las 06:00 horas a.m. y 18:00 horas p.m. para ambos años, siendo el promedio de I.I. de 26,2 en 2011 en el rango horario mencionado y en 2012 un I.I. de 34,5. Figura 2.

En el año 2011 se declararon en Ecuador un total de 8.301 (88,9\%) incapacidades de carácter temporal y 13.074 en 2012 (95,7\%), correspondiendo un I.I. de 373,14 y 527,02 para cada año respectivo. Tabla 4. 
Tabla 4.

Distribución de I.I. Tipo de incapacidad 2011-2012.

\begin{tabular}{|c|c|c|c|c|c|c|}
\hline $\begin{array}{l}\text { TIPO DE } \\
\text { INCAPACIDAD }\end{array}$ & n A.T. & $\begin{array}{c}2011 \\
\% \text { n A.T. }\end{array}$ & I.I. & n A.T. & $\begin{array}{c}2012 \\
\% \text { n A.T. }\end{array}$ & I.I. \\
\hline Temporal & 8301 & $88,9 \%$ & 373,14 & 13074 & $95,7 \%$ & 527,02 \\
\hline Permanente Parcial & 710 & $7,6 \%$ & 31,92 & 280 & $2,1 \%$ & 11,29 \\
\hline Permanente Total & 31 & $0,3 \%$ & 1,39 & 7 & $0,1 \%$ & 0,28 \\
\hline Permanente Absoluta & 22 & $0,2 \%$ & 0,99 & 13 & $0,1 \%$ & 0,52 \\
\hline Muerte & 274 & $2,9 \%$ & 12,32 & 276 & $2,0 \%$ & 11,13 \\
\hline Sin información & 0 & $0,0 \%$ & 0,00 & 7 & $0,1 \%$ & 0,28 \\
\hline TOTAL & 9338 & $100 \%$ & 419,76 & 13657 & $100 \%$ & 550,53 \\
\hline
\end{tabular}

A.T.: accidentes de trabajo I.I.: índice de incidencia

\section{DISCUSIÓN}

De los datos analizados existen razones para creer que en Ecuador la notificación de accidentes de trabajo por parte del empleador es escasa en comparación con el resto de países latinoamericanos. ${ }^{11}$ Si bien se incrementó el número de notificaciones al Instituto Ecuatoriano de Seguridad Social de 2011 a 2012, no se trata de una situación alarmante en cuanto a las condiciones de seguridad y salud de los trabajadores en los centros de trabajo, sino más bien, a la entrada en vigor en 2011 de la Resolución No. C.D. 3907, vigente hasta el día de hoy, donde se establecen los plazos de notificación de los accidentes de trabajo por parte del empleador. Todo ello unido a las campañas de divulgación y sensibilización mediantes congresos, charlas y talleres por la Dirección Nacional del Seguro General de Riesgos del Trabajo.

Sería interesante establecer un modelo de Clasificación Nacional de Actividades Económicas ${ }^{13}$ para el registro oficial de los accidentes de trabajo y de enfermedades. Si bien la industria manufacturera ha experimentado un aumento en la notificación al organismo oficial correspondiente, este hecho se debe a que existen en la actualidad un gran número de empresas en el país de este tipo $\mathrm{y}$ es donde mayor número de trabajadores laboran ${ }^{15}$; a su vez, esta actividad se encuadra como una actividad de alto riesgo donde las consecuencias de los factores de riesgo ocupacional a la salud son más graves. ${ }^{16-17}$

Los hombres, respecto a las mujeres, son los que mayoritariamente sufren accidentes de trabajo debido principalmente a que las actividades que desarrollan entrañan mayor nivel de riesgo laboral.

Las accidentes in-itínere (los que sufre el trabajador al ir y volver de su domicilio al lugar de trabajo) son de gran relevancia por las consecuencias en términos de daño o lesión, sería una línea de investigación futura el poder tratar este tema con mayor profundidad.

La actividad económica con mayor siniestralidad laboral es la manufactura (mayoritariamente se emplea maquinaria y en ciertas tareas el trabajo manual); se asocia a la ubicación de la lesión, predominando las lesiones en miembros inferiores y superiores. No disponiendo de datos oficiales y válidos sobre la distribución de la población afiliada poractividad económica se dificultó el análisis y cálculo del índice de incidencia.

Se concluye que las notificaciones de accidentes de trabajo y enfermedades profesionales en Ecuador siguen siendo escasas respecto a otros países vecinos como Colombia ${ }^{18}$ (401.749 accidentes de trabajo en 2011 y 2012 con 388.008) y Perú ${ }^{19}$ (16.640 en 2012).

\section{CONCLUSIONES}

Este trabajo permite establecer una aproximación sobre la siniestralidad laboral en Ecuador $\mathrm{y}$, a su vez, la comparación entre países. Las notificaciones de accidentes de trabajo registrados en 2012 representan una incidencia de 550,53 casos por cada 100.000 trabajadores, indice superior al registrado en 2011, con 419,76.

En 2012 la provincia de Cañar es la de mayor incidencia en accidentes de trabajo con 1913.29 casos por cada 100.000 trabajadores, seguida de Napo con 1215,17 . Por lo que respecta a las principales provincias del país, Pichincha se sitúa en 371,17 casos y 1037,75 la provincia del Guayas en este mismo año.

El lugar de trabajo es el de mayor índice de incidencia respecto a los in-itínere y comisión-servicio para ambos períodos de 2011 y 2012.

Los accidentes de trabajo con lesión en los miembros superiores son los de mayor incidencia, 196,4 por cada 100.000 trabajadores, seguida por las lesiones en miembros inferiores con 158,8 para el 2012, datos que no varian proporcionalmente en el año anterior.

Respecto a las consecuencias derivadas por los accidentes de trabajo, las fracturas y luxaciones, torceduras y esguinces, conmociones y traumatismos internos, amputaciones, traumatismos superficiales, contusiones y aplastamientos y otras heridas son las de mayor incidencia.

La incidencia de los accidentes de trabajo según el día de la semana es muy semejante a lo largo de la semana (lunes a viernes), no existiendo diferencias significativas, siendo de menor incidencia los domingos. De igual modo, ocurre con las horas del día en las que con mayor frecuenciase registraron los accidentes. Las 10:00 h. a.m. es la de mayor incidencia:se producen 57,7 accidentes de trabajo a las 10 de la mañana por cada 100.000 trabajadores.

Por último, la incidencia de los accidentes de trabajo que derivan en una incapacidad temporal en 2012 es de 527,02 por cada 100.000 trabajadores. 


\section{REFERENCIAS}

1. OISS-CSO. Organización Iberoamericana de Seguridad Social-Consejo de Salud Ocupacional. Sistema armonizado de indicadores básicos de siniestralidad y salud laboral en Iberoamérica. Estrategia Iberoamericana de Seguridad y Salud en el Trabajo 2010-2013. San José: OISS-CSO; 2013.

2. España. Instituto Nacional de Seguridad e Higiene en el Trabajo. Siniestralidad laboral: periodo julio 2011-junio 2012. Madrid: INSHT, 2012.

3. OIT. Informe III. Estadisticas de lesiones profesionales. Decimosexta Conferencia Internacional de Estadísticos del Trabajo; 6-15 oct 1998, Ginebra, Suiza. Ginebra: OIT; 1999.

4. Leigh JP, Markowitz SB, Fahs M, Shin C, Landrigan PJ. Occupational injury and illness in the United States. Estimates of costs, morbidity and mortality. Arch Intern Med. 1997;157:1557-1568.

5. Kraut, A. Estimates of the extent of morbidity and mortality due to occupational diseases in Canada. Am J IndMed. 1994;25:267-78

6. Ecuador. Instituto Ecuatoriano de Seguridad Social. Resolución C.D. No. 333; Reglamento para el Sistema de Auditoria de Riesgos del Trabajo- SART. Quito: Instituto Ecuatoriano de Seguridad Social; 2010.

7. Ecuador. Instituto Ecuatoriano de Seguridad Social .Resolución C.D. No. 390 Reglamento del Seguro General de Riesgos del Trabajo. Quito: Instituto Ecuatoriano de Seguridad Social; 2011.

8. Ecuador. Ministerio del Trabajo. Decreto Ejecutivo 2393 Reglamento de Seguridad y Salud de los Trabajadores y Mejoramiento del Medio Ambiente de Trabajo,. Quito: Ministerio del Trabajo; 1986.

9. Ecuador. Ministerio del Trabajo y Seguridad Social-Instituto Ecuatoriano de Seguridad Social. Acuerdo No. 1404 Reglamento para el Funcionamiento de los Servicios Médicos de Empresa. Quito: MTSS-IESS; 1978.

10. Ecuador. Asamblea Constituyente de la República. Constitución de la República del Ecuador. Montecristi: Asamblea Nacional Constituyente; 2008.

11. OISS-Organización Iberoamericana de Seguridad Social. Recopilación de los principales indicadores de siniestralidad laboral y enfermedad ocupacional utilizados en Iberoamérica; Prospección realizada en Internet. Madrid: OISS; 2012 [citado jul 2014]. Disponible en: http://www.oiss.org/estrategia/IMG/ pdf/Informe_sobre_siniestralidad.pdf

12. Ecuador. Instituto Ecuatoriano de Seguridad Social. Resolución No. 741 de 1990; Reglamento General del Seguro de Riesgos del Trabajo, reformado por la Resolución No. 874 de 1996 y Resolución No. C.I. 113 de 2001. Quito: IESS; 2001.
13. Ecuador. INEC-Instituto Nacional de Estadistica y Censo-Unidad de Análisis de Síntesis. Clasificación Nacional de Actividades Económicas (CIIU REV. 4.0). Quito: INEC; 2012.

14. Ecuador. Instituto Ecuatoriano de Seguridad Social. Proyecto de inteligencia de Negocio [en línea]. Quito: IESS; 2011 [citado 15 jul 2014]. Disponible en: http://www.iess.gob.ec/documents/10162/44151/HL_REPORTE_ ESTADISTICAS_2011-09.pdf.

15. Ecuador. INEC-Instituto Nacional de Estadística y Censo. Encuesta Nacional de Empleo, Desempleo y Subempleo [en línea]. Quito: INEC; 2015 [citado 9 feb 2015]. Disponible en: http://www.ecuadorencifras.gob.ec/censo-nacionaleconomico/.

16. OIT. Organización Internacional del Trabajo. Informe III. Estadísticas de lesiones profesionales. Decimosexta Conferencia Internacional de Estadísticos del Trabajo. 6-15 oct. 1998; Ginebra, Suiza [en línea].Ginebra: OIT; 1999 [citado 15 dic 2014].Disponible en: http://www.lo.org/wcmsp5/groups/public/---dg reports/--stat/documents/meetingdocument/wcms_088377.pdf.

17. OIT. Sistema de gestión de la SST: una herramienta para la mejora continua [en línea]. Ginebra: OIT; 2011 [citado 16 dic 2014]. Disponible en: http:// www.ilo.org/wcmsp5/groups/public/@ed_protect/@protrav/@safework/ documents/publication/wcms_154127.pdf.

18. Colombia. Ministerio del Trabajo. Avances de la Dirección de Riesgos Laborales [en línea]. Bogotá: Ministerio del Trabajo; 2012 [citado 4 mar 2015]. (Boletín de Riesgos Laborales, 5). Disponible en: Boletin $\% 2005 \% 20$ Septiembre $\% 20$ del $\% 20$ 2012\%20.pdf

19. Perú. Ministerio del Trabajo. Boletín estadistico notificaciones de accidentes de trabajo, incidentes peligrosos y enfermedades ocupacionales [en línea]. Lima: Ministerio del Trabajo; 2015. [citado 3 mar 2015]. Disponible en: http:// www.mintra.gob.pe/mostrarContenido.php?id=730\&tip $=9$

\section{Referencia consultada:}

European Commission; Eurostat.EuropeanLabourForceSurvey[on line]. Luxembourg: Eurostat; 2014-[cited 21 jul 2014]. Available on: http://ec.europa.eu/eurostat/ home?p_auth=jAF0fNa6\&p_p_id=estatsearchportlet_WAR_estatsearch portlet\&p_p_lifecycle $=1 \& p \_p \_s t a t e=$ maximized \&p_p_mode $=v i e w \&$ _ estatsearchportlet_WAR_estatsearchportlet_action=search\&text=The+Europe an+Union+labour+force+survey 